\title{
Neural Stem Cells Show Bidirectional Experience-Dependent Plasticity in the Perinatal Mammalian Brain
}

\author{
Tod E. Kippin, ${ }^{1 \star}$ Sean W. Cain, ${ }^{2 \star}$ Zahra Masum, $^{2}$ and Martin R. Ralph ${ }^{2}$ \\ Departments of ${ }^{1}$ Medical Genetics and Microbiology and ${ }^{2}$ Psychology, University of Toronto, Toronto, Ontario M5S 1A8, Canada
}

\begin{abstract}
Many of the effects of prenatal stress on the endocrine function, brain morphology, and behavior in mammals can be reversed by brief sessions of postnatal separation and handling. We have tested the hypothesis that the effects of both the prenatal and postnatal experiences are mediated by negative and positive regulation of neural stem cell (NSC) number during critical stages in neurodevelopment. We used the in vitro clonal neurosphere assay to quantify NSCs in hamsters that had experienced prenatal stress (maternal restraint stress for $2 \mathrm{hr}$ per day, for the last $7 \mathrm{~d}$ of gestation), postnatal handling (maternal- offspring separation for $15 \mathrm{~min}$ per day during postnatal days 1-21), or both. Prenatal stress reduced the number of NSCs derived from the subependyma of the lateral ventricle. The effect was already present at postnatal day 1 and persisted into adulthood (at least 14 months of age). Similarly, prenatal stress reduced in vivo proliferation in the adult subependyma of the lateral ventricle. Conversely, postnatal handling increased NSC number and reversed the effect of prenatal stress. The effects of prenatal stress on NSCs and proliferation and the effect of postnatal handling on NSCs did not differ between male and females. The findings demonstrate that environmental factors can produce changes in NSC number that are present at birth and endure into late adulthood. These changes may underlie some of the behavioral effects produced by prenatal stress and postnatal handling.
\end{abstract}

Key words: prenatal stress; postnatal handling; maternal separation; neurodevelopment; neurosphere; neurons; glia

\section{Introduction}

Environmental stimuli during the perinatal period have profound effects on developmental processes resulting in permanent alterations in nervous system structure and function, and these effects contribute to individual differences in a number of behavioral domains (Meaney, 2001; Andersen, 2003; Maccari et al., 2003; Pryce and Feldon, 2003). Although key mediators of these effects have been identified, the precise mechanistic events mediating the development of these physiological and behavioral alterations remain to be fully elucidated. The developmental stage at which these stimuli most effectively produce permanent changes coincides with the time that neural stem cells (NSCs) are dynamically regulated (Tropepe et al., 1999; Martens et al., 2000). In the present study, we examine the ability of environmental stimuli during the perinatal period to regulate NSCs.

Prenatal stress and brief maternal separation are commonly used and well described models of environmental influences on development and subsequent nervous system function and behavior. Administration of stressful stimuli during late gestation (prenatal stress) produces permanent hyper-reactivity to environmental stressors in the offspring resulting in emotional and cognitive behavioral deficits mediated by alterations in the functional properties of the limbic system and hypothalamic-pitu-

Received Sept. 5, 2003; revised Feb. 4, 2004; accepted Feb. 7, 2004.

The work was supported by a grant from the Natural Sciences and Engineering Research Council of Canada to M.R.R., a Natural Sciences and Engineering Research Council of Canada fellowship awarded to T.E.K., and a Natural Sciences and Engineering Research Council of Canada scholarship awarded to S.W.C.

*T.E.K. and S.W.C. contributed equally to the preparation of this manuscript.

Correspondence should be addressed to Tod Kippin, Medical Genetics and Microbiology, University of Toronto, 1 King's College Circle, MSB 1105, Toronto, Ontario M5S 1A8, Canada. E-mail: tod.kippin@utoronto.ca.

DOI:10.1523/JNEUROSCI.0110-04.2004

Copyright $\odot 2004$ Society for Neuroscience $\quad$ 0270-6474/04/242832-05\$15.00/0 itary-adrenal (HPA) axis (for review, see Kofman, 2002; Maccari et al., 2003). Conversely, brief separation of mother from pups during nursing (postnatal handling) produces permanent hyporeactivity to environmental stressors in the offspring resulting in emotional and cognitive behavioral benefits (under standard laboratory conditions) that are also mediated by alterations in the function of the limbic system and HPA axis (for review, see Levine, 1994; Meaney, 2001; Pryce and Feldon, 2003). Furthermore, the behavioral and neuroendocrine effects of prenatal stress appear to be ameliorated or abolished by subsequent postnatal handling (Wakshlak and Weinstock, 1990; Vallee et al., 1997; Takahashi, 1998), suggesting that common substrates underlie the effects of these manipulations.

Given the dynamic regulation of NSCs coinciding with the effective periods of prenatal stress and postnatal handling effects, we hypothesized that the effects of prenatal stress and postnatal handling may be mediated through effects on NSCs with subsequent effects on neurodevelopment. Consistent with this hypothesis, prenatal stress is associated with reduced neurogenesis in the adult rat hippocampus that correlates with learning deficits on hippocampal-dependent tasks (Lemaire et al., 2000). Recently, we have characterized the behavioral effects of prenatal stress in hamsters and have found learning deficits similar to those of other rodents, as well as disruptions in circadian rhythms and reduced longevity (Cain et al., 2001). Accordingly, we examined the effects of prenatal stress and postnatal handling on NSCs in the developing and adult hamster using the neurosphere assay (Reynolds and Weiss, 1996) as an in vitro index for the functional characteristics of NSC number in vivo (Morshead et al., 2003). Our findings demonstrate that NSC number is regulated bidirectionally by environmental stimuli during the perinatal pe- 
riod, raising the possibility that the effects on NSC number are linked to the pervasive effects of these stimuli.

\section{Materials and Methods}

Subjects. All subjects were Golden hamsters (Mesocricetus auratus) bred at the University of Toronto from stock derived from Charles River Canada (Montreal, Quebec). Animals were group housed with littermates $(n=3-6$ per cage $)$ in polypropylene cages $(22 \times 44 \mathrm{~cm})$ with ad libitum access to food and water in a colony room maintained on a 14/10 $\mathrm{hr}$ light/dark cycle. In all experiments, pups were weaned and dams killed with carbon dioxide at postnatal day 21 . All pups were then left undisturbed in their home cages except for regular cage maintenance and food and water checks until they were killed to determine NSC numbers.

Prenatal stress treatment. Pregnant dams were randomly assigned to prenatal stress or nonstress (control) conditions (two dams per condition for each age examined). Dams in the control group were left undisturbed throughout pregnancy. Dams in prenatal stress group were subjected to restraint stress from days 9 to 16 of gestation. The restraint stress treatment entailed removing dams from their home cages and confining them in narrow transparent plastic tubes $(11 \mathrm{~cm}$ long $\times 6 \mathrm{~cm}$ diameter) for $2 \mathrm{hr}$ per day. All prenatal stress treatments began $1 \mathrm{hr}$ after lights off in dim red light. Offspring from both the prenatal stress and control groups were killed, and the number of NSCs was determined using the neurosphere assay (see below) at postnatal days $1,30,90,240$, and 420 . Separate litters (total of 10 litters per condition) were used for each age, and the prenatal stress and control groups comprised eight animals (four males and four females; two animals per sex per litter) at each age. Additional control and prenatal stress animals were selected from three litters per condition at $\sim 45 \mathrm{~d}$ of age for determination of in vivo proliferation levels using bromodeoxyuridine (BrdU) labeling (see below); total animal numbers for BrdU labeling were for the control group, $n=11$ ( 3 males and 8 females), and for the prenatal stress group, $n=10$ ( 3 males and 7 females).

Postnatal handling treatment. Litters of pups from prenatal stress and nonstress dams were randomly assigned to postnatal handling or nonhandling conditions. Nonhandling dams and litters were left undisturbed except for regular food and water checks until the pups were weaned at $21 \mathrm{~d}$ of age. The postnatal handling treatment was used from postnatal days 1 to 21 . Each day beginning $6 \mathrm{hr}$ after lights on, dams of postnatal handling pups were moved from their home cages to clean, empty cages, and then their pups were moved to small plastic containers lined with wood chip and paper towel bedding. After $15 \mathrm{~min}$, pups were returned to their home cages, followed shortly thereafter by the dams. Dams in the prenatal stress-postnatal handling group were subjected to restraint stress identical to the prenatal stress only group, and after birth their pups were subjected to postnatal handling identical to that of the postnatal handling only group. Offspring from the control (nonstressednonhandled), prenatal stress, postnatal handling, and prenatal stresspostnatal handling groups were killed, and the number of NSCs was determined using the neurosphere assay. Subjects were derived from five to seven litters per group with a maximum of three to four offspring per litter of approximately equal numbers of males and females from each litter. The total numbers for each group were as follows: control, $n=28$ (12 males and 16 females); prenatal stress, $n=20$ (10 males and 10 females); postnatal handling, $n=20$ (9 males and 11 females); prenatal stress-postnatal handling, $n=14$ ( 6 males and 8 females).

Repeated adult stress treatment. Adult (6-8 weeks of age) male and nonpregnant female hamsters received either daily restraint stress for 2 $\mathrm{hr} / \mathrm{d}$ for $21 \mathrm{~d}$ using the same procedure used to induce prenatal stress $(n=6)$ or no treatment $(n=7)$. One day after the final treatment, all animals were killed, and the number of NSCs was determined using the neurosphere assay.

Neurosphere assay. The number of NSCs in vivo was determined using the neurosphere assay, a tissue culture assay that assesses the functional properties of proliferation, self-renewal, and multipotentiality possessed by NSCs. The subependyma was dissected as described by Reynolds and Weiss (1996). Subependyma tissue was subjected to enzymatic digestion $(1.33 \mathrm{mg} / \mathrm{ml}$ trypsin, $0.67 \mathrm{mg} / \mathrm{ml}$ hyaluronidase, and $0.2 \mathrm{mg} / \mathrm{ml}$ kynurenic acid) for $50 \mathrm{~min}$ at $37^{\circ} \mathrm{C}$ and then isolated in serum-free media (SFM) with trypsin inhibitor. Next, the tissue was mechanically dissoci- ated into a single-cell suspension with a small-bore, fire-polished Pasteur pipette. Cell density was determined using trypan blue exclusion.

Cells were cultured in the neurosphere assay under clonal conditions (Morshead et al., 2003). Cells were plated at 20 cells per microliter in 24 -well ( $0.5 \mathrm{ml}$ per well) uncoated plates (Nunclon) in serum-free medium containing $20 \mathrm{ng} / \mathrm{ml}$ epidermal growth factor (EGF; mouse submaxillary; Sigma, St. Louis, MO), $10 \mathrm{ng} / \mathrm{ml}$ fibroblast growth factor-2 (FGF; human recombinant; Sigma), and $2 \mu \mathrm{g} / \mathrm{ml}$ heparin (Sigma). The total number of spheres that formed in each well was counted after $7 \mathrm{~d}$. Under these conditions it has been shown that sphere colonies are derived from single cells and serve as an index of the number of in vivo NSCs (Morshead et al., 2003). To determine the in vitro self-renewal capacity, spheres in bulk were dissociated into single-cell suspension and recultured under the same conditions as primary cultures, and the number of secondary spheres was determined after $7 \mathrm{~d}$. To determine multipotentiality, single spheres were differentiated in 24 -well plates coated with MATRIGEL basement membrane matrix $(0.6 \mathrm{mg} / \mathrm{ml}$ in SFM; Becton Dickinson, Mississauga, Ontario) containing $0.5 \mathrm{ml}$ of SFM containing $1 \%$ fetal bovine serum (Invitrogen, Gaithersburg, MD). Wells were processed $7 \mathrm{~d}$ later using immunocytochemistry as described previously (Seaberg and van der Kooy, 2002). We used anti- $\beta$-tubulin isotype III mouse monoclonal IgG (1:500; Sigma), anti-GFAP rabbit polyclonal IgG (1:400; Biomedical Technologies, Stoughton, MA), FITC goat antirabbit (1:200; Jackson ImmunoResearch, West Grove, PA), and TRITC goat anti-mouse (1:200; Jackson ImmunoResearch). Cells were counterlabeled with the nuclear stain Hoechst 33258 ( $1 \mu \mathrm{g} / \mathrm{ml}$; Sigma). Fluorescence was visualized using a Nikon Microphot microscope, and the presence of neurons and astrocytes was confirmed by $\beta$-tubulin isotype $\mathrm{III}^{+}$ and $\mathrm{GFAP}^{+}$cells, respectively.

Bromodeoxyuridine labeling and detection. Bromodeoxyuridine labeling of mitotic cells in S phase was used to determine the effect of prenatal stress on in vivo cellular proliferation in the subependyma of the lateral ventricle. Animals were injected with $\mathrm{BrdU}$ (Sigma) $(60 \mathrm{mg} / \mathrm{kg}$, i.p., in saline) every $3 \mathrm{hr}$ for five injections and killed $24 \mathrm{hr}$ after the final injection. Animals were overdosed with pentobarbital and perfused transcardially with PBS and $4 \%$ paraformaldehyde. Brains were removed and postfixed overnight, cryoprotected with $30 \%$ sucrose in PBS at $4^{\circ} \mathrm{C}$, and then sectioned at $20 \mu \mathrm{m}$ thickness on a cryostat. Sections were incubated in $1 \mathrm{~N} \mathrm{HCl}$ at $60^{\circ} \mathrm{C}$ for $30 \mathrm{~min}$, rinsed in PBS, and subsequently incubated in rat anti-BrdU antibody ( $1: 100$; Chemicon, Temecula, CA) at $4^{\circ} \mathrm{C}$ overnight, followed by FITC donkey anti-rat antibody (1:200; Jackson ImmunoResearch). The total number of BrdU-labeled cells in the subependyma was estimated using the optical dissector method (Coggeshall and Lekan, 1996) in every 10th section (approximately every $200 \mu \mathrm{m}$ ) from the rostral tip of the crossing of the corpus callosum rostrally and extending caudally to the rostral tip of the crossing of the anterior commissure. The average number of BrdU-labeled cells per section (i.e., combined for subependyma of the left and right lateral ventricle) was calculated to index in vivo proliferation.

\section{Results}

\section{Prenatal stress permanently decreases NSC number in the subependyma of the lateral ventricle}

To investigate the effect of prenatal stress on the regulation of NSC, pregnant hamsters received either restraint stress for the final $7 \mathrm{~d}$ of pregnancy or no treatment, and the number of NSCs was assessed using the clonal neurosphere assay in the offspring at postnatal days $1,30,90,240$, and 420. Prenatal stress decreased the number of neurospheres derived from the subependyma of the lateral ventricle in offspring at all ages tested (Fig. 1). Twoway (group $\times$ age) ANOVA revealed a significant effect of group $\left(F_{(1,70)}=19.05 ; p<0.0001\right)$, with prenatal stress hamsters having decreased neurospheres relative to nonstressed ones at all ages (all $p<0.05)$. There was also a significant effect of age $\left(F_{(4,70)}=\right.$ 5.01; $p=0.001)$, with postnatal day 1 hamsters having more neurospheres than all other ages (all $p<0.05$ ) and postnatal day 420 hamsters having fewer neurospheres than all other ages (all $p<$ 


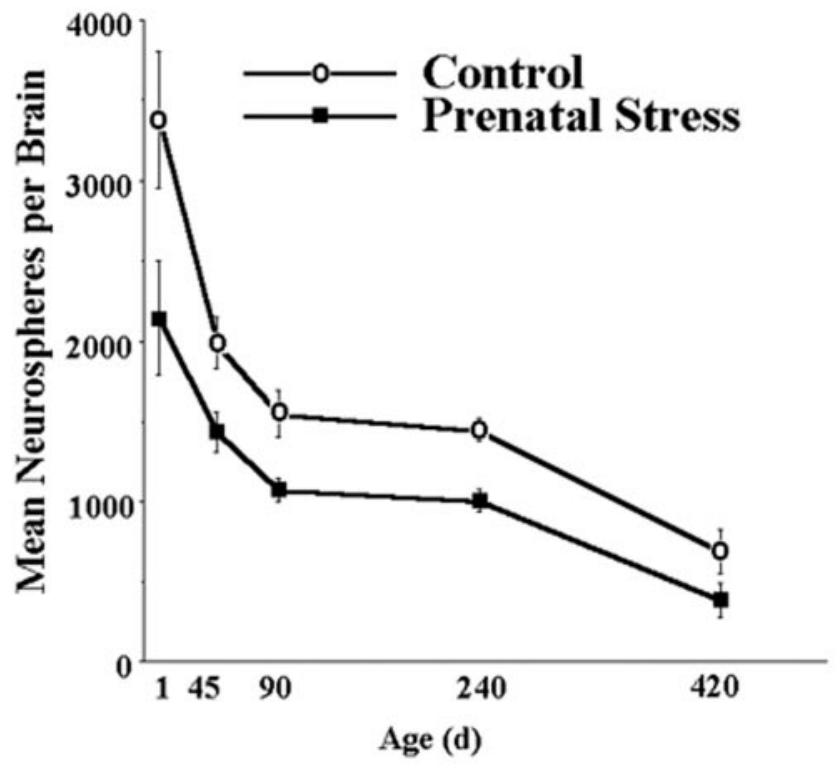

Figure 1. Prenatal stress decreases the number of NSCs derived from the subependyma of the lateral ventricle in both male and female hamsters. The effect is present at postnatal day 1 and persists into advanced ( $420 \mathrm{~d}$ of age) age. Groups differed significantly at each time point.

0.05); however, the interaction between group and age failed to reach statistical significance $\left(F_{(4,70)}=1.03 ; p<0.05\right)$. Notably, prenatal stress decreased the number of neurospheres at postnatal day $1(p<$ $0.05)$, demonstrating that this effect was not caused by postnatal dam-pup or pup-environment interactions. Similarly, the prenatal stress reduction of neurospheres endures until at least $420 \mathrm{~d}$ of age $(p<0.05)$, suggesting that the effect is permanent and additive, with aging declines in the number of NSCs. Further analyses failed to reveal significant effects of $\operatorname{sex}\left(F_{(1,60)}=0.81 ; p>0.05\right)$, interaction between sex and group $\left(F_{(1,60)}=0.05 ; p>0.05\right)$, interaction between sex and age $\left(F_{(4,60)}=0.96 ; p>0.05\right)$, or interaction between sex, group, and age $\left(F_{(4,60)}=0.16 ; p>0.05\right)$. These results indicate that prenatal stress produces a decrease in the number of NSCs that is present at birth and maintained until at least 14 months of age in both male and female hamsters.

Although prenatal stress decreased the number of primary neurospheres derived from the developing and adult brain, it did not produce changes in the characteristics of the NSCs. NSCs derived from control and prenatal stress animals did not differ in terms of their self-renewal or expansion characteristics or multipotentiality, as demonstrated by no effect of prenatal treatment on either in vitro passaging (number of secondary neurospheres) or differentiation (proportion of neurospheres giving rise to neurons and astrocytes) of primary neurospheres from 90-d-old animals (data not shown).

Prenatal stress decreases in vivo proliferation in the adult subependyma of the lateral ventricle

To investigate the effect of prenatal stress on in vivo proliferation, pregnant hamsters received either restraint stress for the final $7 \mathrm{~d}$ of pregnancy or no treatment, and the number of proliferating cells in the subependyma of the lateral ventricle of the offspring ( $\sim 45 \mathrm{~d}$ of age $)$ was determined using BrdU labeling. The number of BrdU-labeled cells in the subependyma of the lateral ventricle was decreased in prenatal stress $(102.10 \pm 12.22)$ relative to control $(140.22 \pm 13.50)$ animals. Two-way (group $\times$ sex $)$ ANOVA revealed a significant effect of group $\left(F_{(1,17)}=6.19 ; p<0.05\right)$, with prenatal stress hamsters having decreased BrdU labeling

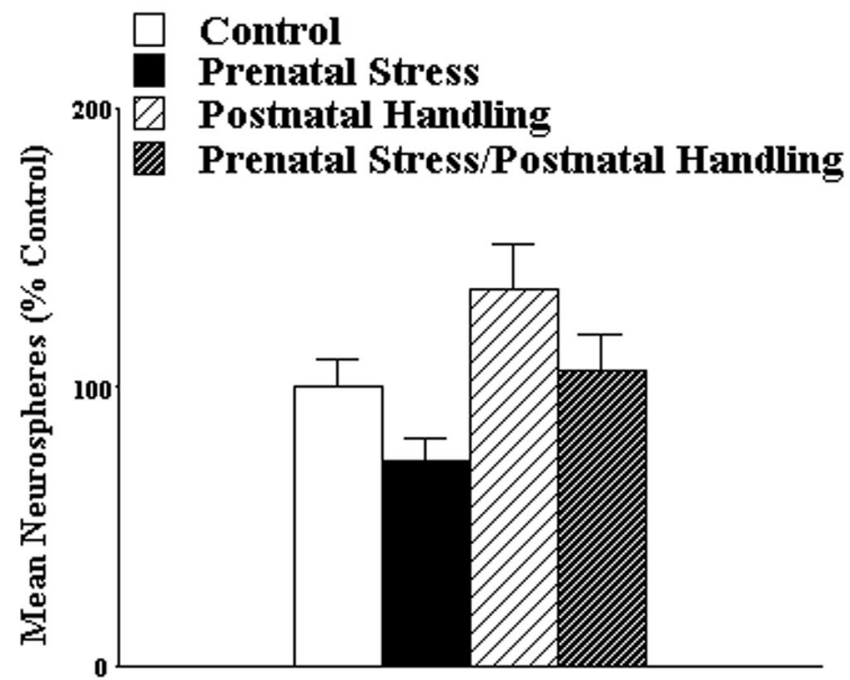

Figure 2. Postnatal handling increases the number of NSCs derived from the subependyma of the lateral ventricle and reverses the effect of prenatal stress at $45 \mathrm{~d}$ of age in both male and female hamsters. ${ }^{*} p<0.05$ for comparison with control group.

relative to nonstressed. Analyses failed to reveal an effect of sex $\left(F_{(1,17)}=0.16 ; p>0.05\right)$ or an interaction between sex and group $\left(F_{(1,17)}=0.10 ; p>0.05\right)$. These findings demonstrate that, similar to the effect on NSCs, prenatal stress produces an enduring reduction in in vivo proliferation in the adult subependyma of the lateral ventricles of both male and female hamsters.

Postnatal handling increases the number of NSCs and reverses the prenatal stress-induced reduction in NSCs in the subependyma of the lateral ventricle

Given that brief postnatal handling produces many effects opposite to those of prenatal stress and is able to reverse many of the effects of prenatal stress, we predicted that postnatal handling alone would increase the number of NSCs and reverse the reduction in NSC number produced by prenatal stress. Pregnant dams and their offspring that had been subjected to prenatal stress or no treatment received either brief ( $15 \mathrm{~min}$ ) daily separation of dam from offspring until weaning (postnatal handling) or were left undisturbed until weaning (control). At $\sim 45 \mathrm{~d}$ of age the number of NSCs in the offspring was assessed using the clonal neurosphere assay. Consistent with the findings of the previous experiment, prenatal stress reduced the number of neurospheres derived from the subependyma of the lateral ventricle. Moreover, postnatal handling increased the number of neurospheres derived from the subependyma of the lateral ventricle and reversed the effects of prenatal stress (Fig. 2). Data are expressed as percentage of control values for each of three separate experiments to reduce between-experiment variability [overall, means \pm SEMs neurospheres were $1398 \pm 274(n=28)$; for control groups in each experiments, they were $1317 \pm 174(n=12), 730 \pm 229$ $(n=8)$, and $2188 \pm 159(n=8)]$. The significance of these effects was confirmed by one-way ANOVA $\left(F_{(3,78)}=8.07 ; p<0.05\right)$. Follow-up analyses using the Tukey method revealed that the prenatal stress group had significantly fewer neurospheres than the other three groups (all $p<0.05$ ) and the postnatal handling alone group had significantly more neurospheres than the control group ( $p<0.05)$, but no other groups differed significantly. Additional analyses failed to reveal a significant effect of sex $\left(F_{(1,74)}=0.67 ; p>0.05\right)$ or an interaction between sex and group $\left(F_{(3,74)}=0.55 ; p>0.05\right)$. These results indicate that prenatal 
stress produces a decrease and postnatal handling produces an increase in the number of NSCs in both male and female hamsters and that these effects are additive.

Although postnatal handling increased the number of primary neurospheres derived from the developing and adult brain, it did not produce changes in the characteristics of the NSCs. NSCs derived from control and postnatal handling animals did not differ in terms of their self-renewal or expansion characteristics or multipotentiality, as demonstrated by no effect of postnatal treatment on either in vitro passaging (number of secondary neurospheres) or differentiation (proportion of neurospheres giving rise to neurons and astrocytes) of primary neurospheres from 90-d-old animals (data not shown).

\section{Repeated adult stress does not alter NSC number in the subependyma of the lateral ventricle}

Given that restraint stress in dams during the prenatal period reduces the number of NSCs in offspring, we examined the effect of daily restraint stress for $21 \mathrm{~d}$ in adult hamsters on the regulation of NSCs. After treatment, no difference was found between the numbers of neurospheres derived from the subependyma of the lateral ventricle of the control $(1665 \pm 127)$ and adult stressed $(1768 \pm 157)$ hamsters. The statistical significance of this observation was confirmed using Student's $t$ test $\left(t_{(11)}=1.26 ; p>\right.$ $0.05)$. These findings demonstrate that restraint stress does not influence the number of NSCs in the adult brain.

\section{Discussion}

Our results demonstrate that environmental experiences in the perinatal period can alter NSC numbers in the mammalian forebrain and that these effects persist into adulthood and appear to be permanent. The offspring of pregnant hamsters receiving daily restraint stress during gestation had decreased NSC numbers (as indexed by the neurosphere assay) at birth and up to 14 months of age (Fig. 1). Hamsters experiencing daily brief separation from their mothers for the first $21 \mathrm{~d}$ of life had increased NSC numbers at 6 weeks of age (Fig. 2). Conversely, repeated daily restraint stress in adult hamsters failed to alter NSC numbers, indicating that the effects of stress are specific to critical periods during development. These findings extend our understanding of the regulation of NSCs beyond the effects of growth factor and pharmacologic manipulations to include environmental stimuli via physiological processes (Fig. 3).

In the adult brain, NSCs give rise to progenitors that migrate and differentiate into new neurons and glia in the olfactory bulb (Luskin, 1993; Lois and Alvarez-Buylla, 1994) and the dentate gyrus of the hippocampus (Taupin and Gage, 2002). Neurogenesis has been shown to be important for the functional properties of both the olfactory bulb (Gheusi et al., 2000; Rochefort et al., 2002) and the hippocampus (Shors et al., 2001, 2002). Lemaire and colleagues (2000) found that prenatal stress-induced learning impairments are associated with an enduring reduction in NSC progeny (i.e., progenitor cells and neurogenesis) in the hippocampus. Similarly, prenatal stress results in olfactory impairments (Marchlewska-Koj et al., 2003), and the present findings demonstrate that prenatal stress produces an enduring reduction in the number of progenitors cells that contribute to the rostral migratory stream in adulthood. These findings suggest that the reduction in NSC number mediates some behavioral effects of prenatal stress. Although no studies have assessed the effects of postnatal handling on adult neurogenesis, postnatal handling has protective effects against hippocampal-dependent learning impairments observed during aging (Meaney et al., 1988, 1991).

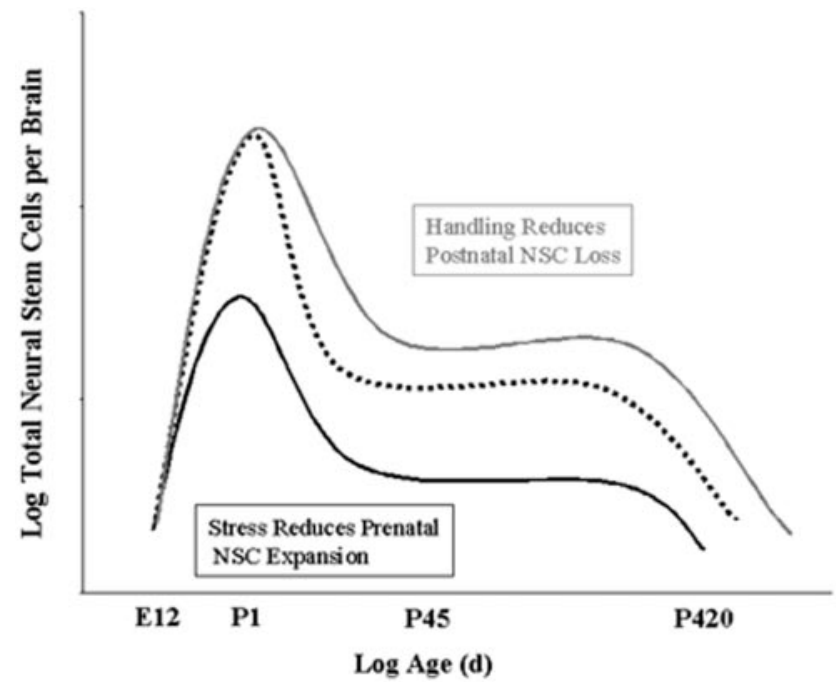

Figure 3. Illustrative summary of effects. Prenatal stress reduces expansion of NSCs between embryonic day 12 (E12) and birth (postnatal day 1; P1). Postnatal handling reduces loss of NSCs between P1 and adulthood. During adulthood, NSC number is stable until advanced age, when numbers decline. Experience-dependent plasticity of NSC population is additive with intrinsic regulation.

Together, this finding and the reduction in NSCs observed during aging suggest that postnatal handling may protect against NSC loss, and this may underlie protection against learning impairments during aging.

The reduction of NSCs produced by prenatal stress may be mediated by glucocorticoids that underlie many prenatal stress effects on neuroendocrine development. Glucocorticoids in the fetal brain are elevated during gestational stress (Lephart et al., 1997). Adrenalectomy of the mother blocks gestational stressinduced alterations in responses to stress in offspring in adulthood (Barbazanges et al., 1996). Administration of exogenous glucocorticoids during gestation produces many of the same the effects as prenatal stress (for review, see Maccari et al., 2003). Although the effects of glucocorticoids on NSCs have not been studied directly, both the amount of progenitor cells (progeny of NSCs) and neurogenesis in the hippocampus are reduced by elevated glucocorticoids produced by stress or exogenous administration (for review, see Gould and Gross, 2002). Conversely, we found that NSC number is not altered by repeated stress in adulthood. Thus, it appears that elevation of glucocorticoids produced by prenatal stress reduces NSC number by inhibiting embryonic expansion. Given this temporal specificity, it is unlikely that glucocorticoids have a general toxic effect on NSCs. In addition, adult stress and glucocorticoid administration act indirectly on hippocampal progenitor proliferation and neurogenesis via modulating glutamate transmission (Gould et al., 1997), and perhaps prenatal stress-induced inhibition of NSC expansion is mediated by glutamate; however, the effects of glutamate on NSCs are unknown. Stress responses also include glutamate-mediated elevation of brain dopamine levels (Zigmond et al., 1998). Dopamine has an inhibitory effect on NSC proliferation (T. E. Kippin, S. Kapur, and D. Van Der Kooy, unpublished observations), and dopamine innervation of the embryonic germinal zone (Ohtani et al., 2003) coincides with the time of prenatal stress-induced reductions of NSCs, suggesting that dopamine may mediate the prenatal stress reduction of NSCs.

The increase in NSC number produced by postnatal handling may be mediated by the modulation of serotonin (5-HT) and neurotrophins, which are important for other neurodevelopmental ef- 
fects of postnatal handling. Meaney and colleagues (for review, see Weaver et al., 2001) have demonstrated that thyroid-dependent modulation of serotonin acting on $5-\mathrm{HT}_{2 \mathrm{~A}}$ and $5-\mathrm{HT}_{7}$ receptors is responsible for the postnatal handling-induced alteration in hippocampal glucocorticoid receptors that underlies the feedback of endocrine responses to stress. Serotonin also regulates progenitor cells and neurogenesis in the hippocampus (for review, see Duman et al., 2001). Together, the postnatal decline in NSC number (Fig. 3) and the effect of serotonin on proliferating cells in the adult brain suggest that postnatal handling may increase the survival or maintain expansion of NSCs in the postnatal brain. However, chronic administration of the serotonin reuptake inhibitor fluoxetine does not increase NSC number in the adult brain (our unpublished observations). Furthermore, high maternal licking-grooming of pups increases survival but not proliferation of neural progenitors in the perinatal hippocampus (Bredy et al., 2003). This suggests that survival, and not proliferation, mediates the effects of postnatal handling on NSCs. Similar to serotonin, nerve growth factor increases during postnatal handling (Cirulli et al., 2000), and it increases the survival of numerous nervous system cells, including neural progenitors (Ostenfeld and Svendsen, 2003). Thus, a potential mechanism of postnatal handling-induced increase in NSC number is the prevention of cell loss.

In summary, the environmental stimuli involved in prenatal stress and postnatal handling manipulations produce opposing and permanent effects on NSC number during a critical perinatal period. Despite our limited knowledge of the roles that NSCs play in nervous system function, there is evidence that changes in NSC number may mediate the expression of the behavioral and endocrine effects produced by early environmental manipulations. Further understanding of the contributions that changes in NSC function make to neuroendocrine physiology will provide insights into the mechanisms mediating the role of environmental factors in psychiatric and neurological disorders.

\section{References}

Andersen SL (2003) Trajectories of brain development: point of vulnerability or window of opportunity? Neurosci Biobehav Rev 27:3-18.

Barbazanges A, Piazza PV, Le Moal M, Maccari S (1996) Maternal glucocorticoid secretion mediates long-term effects of prenatal stress. J Neurosci 16:3943-3949.

Bredy TW, Grant RJ, Champagne DL, Meaney MJ (2003) Maternal care influences neuronal survival in the hippocampus of the rat. Eur J Neurosci 18:2903-2909.

Cain SW, Seco P, Ralph MR (2001) Prenatal stress affects rhythms, learning and longevity in female Syrian hamsters. Abstract presented at VI Latin American Symposium on Chronobiology Natal, Brazil, May.

Cirulli F, Alleva E, Antonelli A, Aloe L (2000) NGF expression in the developing rat brain: effects of maternal separation. Dev Brain Res 123:129-134.

Coggeshall RE, Lekan HA (1996) Methods for determining numbers of cells and synapses: a case for more uniform standards of review. J Comp Neurol 364:6-15.

Duman RS, Nakagawa S, Malberg J (2001) Regulation of adult neurogenesis by antidepressant treatment. Neuropsychopharmacology 25:836-844.

Gheusi G, Cremer H, McLean H, Chazal G, Vincent JD, Lledo PM (2000) Importance of newly generated neurons in the adult olfactory bulb for odor discrimination. Proc Natl Acad Sci USA 97:1823-1828.

Gould E, Gross CG (2002) Neurogenesis in adult mammals: some progress and problems. J Neurosci 22:619-623.

Gould E, Tanapat P, Cameron HA (1997) Adrenal steroids suppress granule cell death in the developing dentate gyrus through an NMDA receptordependent mechanism. Dev Brain Res 103:91-93.

Kofman O (2002) The role of prenatal stress in the etiology of developmental behavioural disorders. Neurosci Biobehav Rev 26:457-470.

Lemaire V, Koehl M, Le Moal M, Abrous DN (2000) Prenatal stress produces learning deficits associated with an inhibition of neurogenesis in the hippocampus. Proc Natl Acad Sci USA 97:11032-11037.

Lephart ED, Watson MA, Jacobson NA, Rhees RW, Ladle DR (1997) Calbindin-D28k is regulated by adrenal steroids in hypothalamic tissue during prenatal development. Dev Brain Res 100:117-120.
Levine S (1994) The ontogeny of the hypothalamic-pituitary-adrenal axis: the influence of maternal factors. Ann NY Acad Sci 746:275-293.

Lois C, Alvarez-Buylla A (1994) Long-distance neuronal migration in the adult mammalian brain. Science 264:1145-1148.

Luskin MB (1993) Restricted proliferation and migration of postnatally generated neurons derived from the forebrain subventricular zone. Neuron 11:173-189.

Maccari S, Darnaudery M, Morley-Fletcher S, Zuena AR, Cinque C, Van Reeth O (2003) Prenatal stress and long-term consequences: implications of glucocorticoid hormones. Neurosci Biobehav Rev 27:119-127.

Marchlewska-Koj A. Kruczek MC, Kapusta J, Pochron E (2003) Prenatal stress affects the rate of sexual maturation and attractiveness in bank voles. Physiol Behav 79:305-310.

Martens DJ, Tropepe V, van der Kooy D (2000) Separate proliferation kinetics of fibroblast growth factor-responsive and epidermal growth factor-responsive neural stem cells within the embryonic forebrain germinal zone. J Neurosci 20:1085-1095.

Meaney MJ (2001) Maternal care, gene expression, and the transmission of individual differences in stress reactivity across generations. Annu Rev Neurosci 24:1161-1192.

Meaney MJ, Aitken DH, Bhatnagar S, Van Berkel CH, Sapolsky RM (1988) Postnatal handling attenuates neuroendocrine, anatomical, and cognitive impairments related to the aged hippocampus. Science 238:766-768.

Meaney MJ, Aitken DH, Sapolsky RM (1991) Environmental regulation of the adrenocortical stress response in female rats and to the clinical understanding of maternal behavior among its implications for individual differences in aging. Neurobiol Aging 12:31-38.

Morshead CM, Garcia AD, Sofroniew MV, van Der Kooy D (2003) The ablation of glial fibrillary acidic protein-positive cells from the adult central nervous system results in the loss of forebrain neural stem cells but not retinal stem cells. Eur J Neurosci 18:76-84.

Ohtani N, Goto T, Waeber C, Bhide PG (2003) Dopamine modulates cell cycle in the lateral ganglionic eminence. J Neurosci 23:2840-2850.

Ostenfeld T, Svendsen CN (2003) Recent advances in stem cell neurobiology. Adv Tech Stand Neurosurg 28:3-89.

Pryce CR, Feldon J (2003) Long-term neurobehavioural impact of the postnatal environment in rats: manipulations, effects and mediating mechanisms. Neurosci Biobehav Rev 27:57-71.

Reynolds BA, Weiss S (1996) Clonal population analyses demonstrate that an EGF-responsive mammalian embryonic CNS precursor is a stem cell. Dev Biol 175:1-13.

Rochefort C, Gheusi G, Vincent JD, Lledo PM (2002) Enriched odor exposure increases the number of newborn neurons in the adult olfactory bulb and improves odor memory. J Neurosci 22:2679-2689.

Seaberg RM, van der Kooy D (2002) Adult rodent neurogenic regions: the ventricular subependyma contains neural stem cells, but the dentate gyrus contains restricted progenitors. J Neurosci 22:1784-1793.

Shors TJ, Miesegaes G, Beylin A, Zhao M, Rydel T, Gould E (2001) Neurogenesis in the adult is involved in the formation of trace memories. Nature 410:372-376.

Shors TJ, Townsend DA, Zhao M, Kozorovitskiy Y, Gould E (2002) Neurogenesis may relate to some but not all types of hippocampal-dependent learning. Hippocampus 12:578-584.

Takahashi LK (1998) Prenatal stress: consequences of glucocorticoids on hippocampal development and function. Int J Dev Neurosci 16:199-207.

Taupin P, Gage FH (2002) Adult neurogenesis and neural stem cells of the central nervous system in mammals. J Neurosci Res 69:745-749.

Tropepe V,. Sibilia M, Ciruna B, Rossant J, Wagner EF, van der Kooy D (1999) Distinct neural stem cells proliferate in response to EGF and FGF in the developing mouse telencephalon. Dev Biol 208:166-188.

Vallee M, Mayo W, Dellu F, Le Moal M, Simon H, Maccari S (1997) Prenatal stress induces high anxiety and postnatal handling induces low anxiety in adult offspring: correlation with stress-induced corticosterone secretion. J Neurosci 17:2626-2636.

Wakshlak A, Weinstock M (1990) Neonatal handling reverses behavioral abnormalities induced in rats by prenatal stress. Physiol Behav 48:289-292.

Weaver IC, Plante P, Weaver S, Parent A, Sharma S, Diorio J, Chapman KE, Seckl JR, Szyf M, Meaney MJ (2001) Early environmental regulation of hippocampal glucocorticoid receptor gene expression: characterization of intracellular mediators and potential genomic target sites. Mol Cell Endocrinol 185:205-218.

Zigmond MJ, Castro SL, Keefe KA, Abercrombie ED, Sved AF (1998) Role of excitatory amino acids in the regulation of dopamine synthesis and release in the neostriatum. Amino Acids 14:57-62. 